\title{
Mesh-like vascular changes in copper deficiency-induced rat cardiomyopathy
}

\author{
Rina Yamane ${ }^{*}$, Makoto Tanaka1, Nao Kikugawa1, Hideki Yasui1, Kenta Takei, \\ Miwa Harada ${ }^{1}$, and Shinya Kaneda ${ }^{1}$ \\ ${ }^{1}$ Naruto Research Laboratory, Research and Development Center, Otsuka Pharmaceutical Factory, Inc., 115 Kuguhara, Tateiwa, \\ Muya-cho, Naruto, Tokushima 772-8601, Japan
}

\begin{abstract}
The pathological effects of copper deficiency (COD) are well known. However, the pathogenesis of cardiomyopathy resulting from COD remains unclear. In this study, aimed to elucidate the pathogenesis of COD-induced cardiomyopathy by examining the morphology of the cardiovascular system in copper-deficient rats using histopathology, immunohistochemistry, and scanning and transmission electron microscopy. Changes detected in the myocardium and interstitium were consistent with those reported for COD. Morphological changes included mesh-like changes in the capillary endothelial cells that appear to be a novel finding in COD-induced cardiomyopathy. These changes are hypothesized to result from abnormal vascular remodeling following damage to the basement membrane and due to the mechanical effects of myocardial contractions. Although cardiomyopathy may be associated with microcirculatory disorders arising from these lesions, further investigations are necessary to demonstrate a causal relationship between the pathogenesis of cardiomyopathy and the contribution of these lesions to disease progression. (DOI: 10.1293/tox.2020-0029; J Toxicol Pathol 2021; 34: 127-133)
\end{abstract}

Key words: copper deficiency, cardiomyopathy, microcirculation, vascular endothelial cell, rat, basal lamina

The role of copper as an essential trace element includes its ability to act as a dynamic center for enzymes and physiologically active substances and for the expression and maintenance of their functions. Major copper-dependent enzymes include lysyl oxidase, ceruloplasmin, amine oxidase, cytochrome $c$ oxidase, and superoxide dismutase1,2. Copper plays a role in hematopoiesis, bone metabolism, connective tissue metabolism, nervous function, pigment regulation, among other processes. It also exerts important antioxidant effects $^{3}$.

The well-known effects of copper deficiency (COD) in humans include leukopenia, anemia, and osteoporosis 3 . Cardiovascular abnormalities, such as electrocardiogram (ECG) abnormalities, myocardial infarction, and tachycardia, have also been reported in experimental COD 4 , 5. Furthermore, cardiac hypertrophy has been observed in pigs fed a copper-deficient diet; several pigs in the study died from hemopericardium caused by myocardial and aortic rupture 6 . Additionally, several studies have shown increases in absolute heart weight and heart weight to body weight ratio $^{7}$ as

Received: 30 April 2020, Accepted: 3 September 2020

Published online in J-STAGE: 5 October 2020

*Corresponding author: R Yamane (e-mail: Yamane.Rina@otsuka.jp)

(C)2021 The Japanese Society of Toxicologic Pathology

This is an open-access article distributed under the terms of the Creative Commons Attribution Non-Commercial No Derivatives (by-nc-nd) License. (CC-BY-NC-ND 4.0: https:// creativecommons.org/licenses/by-nc-nd/4.0/) well as myocardial hypertrophy ${ }^{8}$ in rats fed a copper-deficient diet from weaning. As with the porcine model, an important cause of death reported in copper-deficient rats was myocardial rupture; fatal hemothorax was also reported ${ }^{9,10}$. Therefore, COD appears to induce cardiovascular diseases and abnormal ECG, including abnormal ST changes ${ }^{10}$, increased QPS amplitude, and prolonged QT intervals ${ }^{11}$. In recent years, many important similarities have been observed between COD in animals and ischemic heart disease in humans, including glucose intolerance, hypercholesterolemia, ECG abnormality, hyperuricemia, and hypertension, all of which are risk factors for ischemic heart disease and are considered to be related to $\mathrm{COD}^{12}$.

Various histopathological changes have been noted in the myocardium of copper-deficient rats, such as focal necrosis of cardiomyocytes under the endocardium ${ }^{7}$ and chronic interstitial inflammation ${ }^{9}$. Two reports examined the intramyocardial capillaries in copper-deficient rat models. In one report, collagen IV immunohistochemical staining demonstrated thickening and distortion of the basement membrane covering the capillaries and cardiomyocytes in rats fed copper-deficient diet for 6-9 weeks from 12 days of age $^{13}$. Another study found thickening and disruption of the basement membrane between cardiomyocytes and capillaries in rats fed copper-deficient diet for 6 weeks during and after weaning ${ }^{14}$. However, neither study reported morphological changes in the capillary endothelial cells.

While previous reports have described the effects of COD on the cardiovascular system, the mechanistic details 
of COD-induced cardiac pathology are largely unknown. In this study, we performed histopathological examination, immunohistochemistry, scanning electron microscopy (SEM), and transmission electron microscopy (TEM) on the hearts of copper-deficient rats to evaluate the morphological changes caused by COD in the cardiovascular system. Herein, we report our novel findings regarding COD-induced myocardial pathogenesis.

The animal experiments were approved by the Animal Experiment Committee of the Otsuka Pharmaceutical Factory, Inc. Ten male Wistar rats (Japan SLC Inc., Shizuoka, Japan) were housed in groups of up to three in a controlled environment $\left(20-26^{\circ} \mathrm{C}\right.$ temperature range, $40-70 \%$ humidity range, 13-16 air changes/h, and $12 \mathrm{~h}$ nominal photoperiod). The animals were fed either normal diet (AIN93G, Oriental Yeast Co., Ltd., Tokyo, Japan, containing $11.4 \mu \mathrm{g}$ $\mathrm{Cu} / \mathrm{g}$ ) or copper-deficient diet (AIN93G copper-deficient diet, Oriental Yeast Co., Ltd., containing $0.3 \mu \mathrm{g} \mathrm{Cu} / \mathrm{g}$ ) for 20 weeks from 6 weeks of age and provided distilled water ad libitum. Clinical signs, body weight, urinalysis, hematology, and blood chemistry were assessed for all the animals. The animals were euthanized at 26 weeks of age by exsanguination under anesthesia. At necropsy, the heart weight was measured, and the body weight-corrected heart weight was calculated.

The heart, liver, kidney, brain, aorta, sternum (bone and bone marrow), duodenum, and sciatic nerve were fixed in $10 \%$ volume by volume $(\mathrm{v} / \mathrm{v})$ neutral buffered formalin, embedded in paraffin, sectioned into $4 \mu \mathrm{m}$ slices, and stained with hematoxylin and eosin (H\&E). Histopathological analysis was performed on all four myocardial chambers of all the animals. Sections of the heart were stained with Masson's trichrome and also immunostained for collagen IV (1:500; Abcam; Cambridge, UK; ab6568) and von Willebrand factor (vWF; 1:1,600; Abcam; ab6994) overnight at $4^{\circ} \mathrm{C}$. For SEM, the heart sections were deparaffinized, labeled using TI-blue small kit (Nisshin EM Co., Ltd., Tokyo, Japan, 335-2), and examined under a scanning electron microscope (FlexSEM 1000; Hitachi High-Technologies Co. Ltd., Tokyo, Japan). Small segments of the heart tissue were collected from near the left ventricle, fixed in phosphate- buffered $2.5 \%$ glutaraldehyde, re-fixed in a phosphate-buffered $1 \%$ osmium tetroxide, embedded in epoxy resin, and ultra-sectioned. These sections were double-stained with uranyl acetate-lead citrate stain and then examined using a transmission electron microscope (H-7800TEM; Hitachi High-Technologies Co., Ltd.).

In the five male Wistar rats fed COD diet for 20 weeks from 6 weeks of age, no gross remarkable changes were noticed at necropsy. The heart weight was $1.19 \pm 0.14 \mathrm{~g}$ (control group: $1.21 \pm 0.10 \mathrm{~g}$ ), and the body weight-corrected heart weight was $0.26 \pm 0.02 \mathrm{~g} / \mathrm{BW} \mathrm{g}$ (control group: $0.24 \pm 0.01$ $\mathrm{g} / \mathrm{BW}$ g). No obvious differences were observed. Hematological examination revealed that the levels of hemoglobin and hematocrit were decreased in rats fed copper-deficient diet relative to those fed normal diet (rats with COD: $12.40 \pm$ $0.75 \mathrm{~g} / \mathrm{dL}$, control: $14.82 \pm 0.53 \mathrm{~g} / \mathrm{dL}$; rats with COD: 34.68 $\pm 1.93 \%$, control: $41.64 \pm 1.05 \%$, respectively). Plasma biochemical examination showed that the levels of $\mathrm{Cu}$ as well as Fe were decreased in rats fed COD diet (rats with COD: $15.00 \pm 4.7 \mu \mathrm{g} / \mathrm{dL}$, control: $156.00 \pm 8.20 \mu \mathrm{g} / \mathrm{dL}$; rats with COD: $107.00 \pm 17.00 \mu \mathrm{g} / \mathrm{dL}$, control: $183.60 \pm 14.9 \mu \mathrm{g} / \mathrm{dL}$, respectively). These changes were attributed to the effect of impaired mobilization of stored iron due to decreased ceruloplasmin activity caused by COD ${ }^{1}$. No other abnormalities were detected in clinical signs, body weight, or urinalysis.

The results of the histopathological examination are summarized in Table 1. Lesions were seen in the area under the left ventricular endocardium in samples from rats fed with copper-deficient diet (Fig. 1A and B), and cardiomyocyte degeneration, necrosis, and mononuclear cell infiltration in the interstitium (Fig. 1E and F) were noted in all cases, whereas fibrosis was observed in two cases. Masson's trichrome staining showed an increase in collagen or reticulum fibers between myocardial cells in the degenerated and necrotic areas (Fig. 1C, D, G, and $\mathrm{H}$ ). These findings are believed to be related to myocardial injury. Interestingly, mesh-like structures of the capillary endothelial cells were observed within the intact, non-degenerative myocardial tissue under the left ventricle (Fig. 2A-D). Compared with that in control samples, immunohistochemical staining showed that collagen IV, a marker for the basement membrane, was

Table 1. Histopathological Findings in Control and Copper-deficient Rats

\begin{tabular}{lccc}
\hline Organ: & Group & Control & Copper-deficient rats \\
\cline { 2 - 4 } Histopathological findings & Number of animals & 5 & 5 \\
\hline Heart & & $<5 / 5>$ & $<0 / 5>$ \\
Degeneration/necrosis, cardiomyocyte, mononuclear cell & \pm & 0 & 3 \\
infiltration, left ventricular endocardium & + & 0 & 2 \\
& $2+$ & 0 & 0 \\
Fibrosis, interstitium, left ventricular endocardium & $3+$ & 0 & 2 \\
& \pm & 0 & 0 \\
Liver, kidney, brain, sciatic nerve, duodenum, aorta, sternum (bone), sternum (marrow) & $+<5 / 5>$ & 0 \\
\hline
\end{tabular}

$<>$ : Not remarkable/Number of animals examined. \pm : slight +:Mild 2+:Moderate 3+: Severe. 


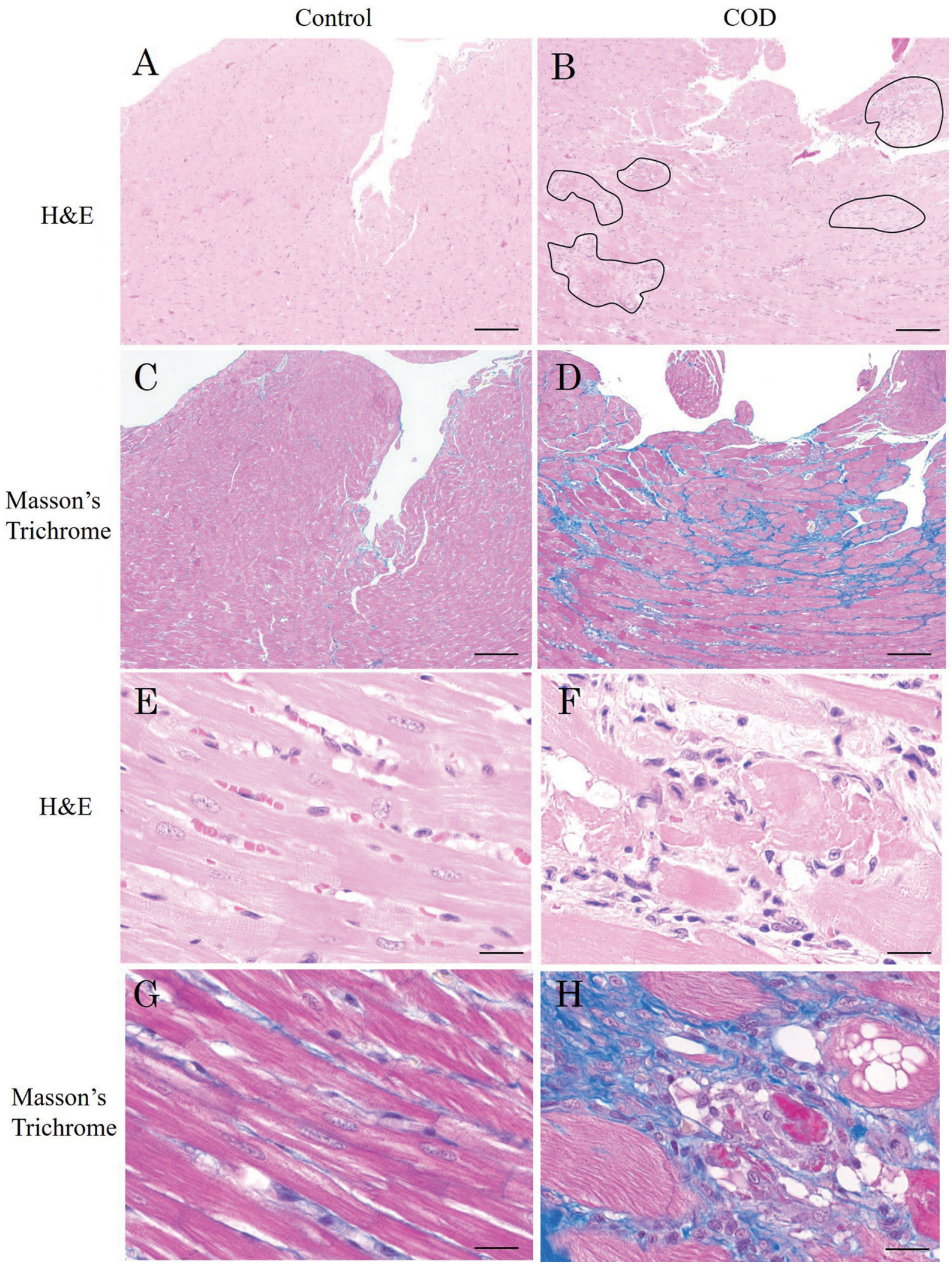

Fig. 1. Light microscopic images of hematoxylin and eosin (H\&E)- (A, B, E, and F) and Masson's trichrome-stained (C, D, G, and H) heart tissue sections from (A, E, C, and G) control rats and (B, F, D and H) rats with copper deficiency (COD). (A, B) Lesions (enclosed by the line) are visible under the left ventricular endocardium in (B) rats with COD. Bar $=200 \mu \mathrm{m}$. (C, D) An increase in the number of collagen or reticulum fibers between the myocardial cells was seen in (D) rats with COD compared to (C) the control rats. Bar $=200 \mu \mathrm{m}$. $(\mathrm{E}, \mathrm{F})$ Cardiomyocyte degeneration, necrosis and mononuclear cell infiltration in the interstitium of $(\mathrm{F})$ rats with COD were observed. Bar $=20$ $\mu \mathrm{m} .(\mathrm{G}, \mathrm{H})$ An increase in collagen or reticulum fibers between the degenerated and necrotic myocardial cells was also observed in $(\mathrm{H})$ rats with COD compared with $(\mathrm{G})$ the control rats. $\mathrm{Bar}=20 \mu \mathrm{m}$. 
either reduced or nonexistent around the myocardial cells under the left ventricular endocardium in specimens from rats fed copper-deficient diet (Fig. 3A-C). This was suggestive of basement membrane injury. Similarly, immunostaining for vWF, a marker for vascular endothelial cells, showed that vascular endothelial cells under the left ventricular endocardium spiked out or thickened to form a mesh-like structure in the lumen of heart tissue specimens from rats with COD compared with that in controls (Fig. 3D-F). The extent of these vascular endothelial changes and myocardial damage correlated with each other. Additionally, disappearance of vascular endothelial cells and infiltration of phagocytic macrophages were observed in the animals with severe myocardial injury (data not shown).

TEM revealed that the basement membrane was detached between the capillaries and myocardial cells. Other findings included projection of capillary endothelial cells into the lumen and thickening of capillary endothelial cells, including mitochondrial dilation (Fig. 4A-C). Furthermore, disappearance of capillary endothelial cells and infiltration of macrophages involving phagosomes were noted in the samples with severe myocardial injury (data not shown). SEM showed that the surface of the capillary endothelium in rats with COD had a roughened appearance and protruded into the lumen in a spike-like or mesh-like manner (Fig. 4D-F).

We performed histopathological examination, immunohistochemistry, SEM, and TEM on the heart of an experimentally induced COD rat model and evaluated the morphological effects of COD on the cardiovascular system. Histopathological examination of hearts from copper-deficient rats revealed degeneration and necrosis of cardiomyocytes under the left ventricular endocardium, mononuclear cell infiltration, fibrosis in the stroma, and mesh-like changes in capillary endothelial cells. Reduced or nonexistent expression of collagen IV, detachment and disappearance of basement membrane, and ultrastructural change/disappearance of vascular endothelial cell suggestive of basement membrane and vascular endothelium damage were observed using immunostaining and electron microscopy. Changes detected in the myocardium and interstitium in this case were similar to those previously reported for $\mathrm{COD}^{7,9}$. In a previous report ${ }^{13}, 14$, no change was observed in the capillary endothelium at the lesion site in a copper-deficient rat model; therefore, these changes constitute a novel finding in our study.

Basement membrane damage was attributed to modification due to mechanical or physical load of the cardiomyocyte-basement membrane under the left ventricular endocardium. Additionally, a decrease in the activity of lysyl oxidase, which is responsible for collagen cross-linking, due to $\mathrm{COD}^{15}$ is thought to play a role in this process. The localization of failure of basement membrane under the left ventricular endocardium and the higher pressure in the left ventricle $^{16}$ further supports this phenomenon during the pathogenesis. Because the changes in distribution of both

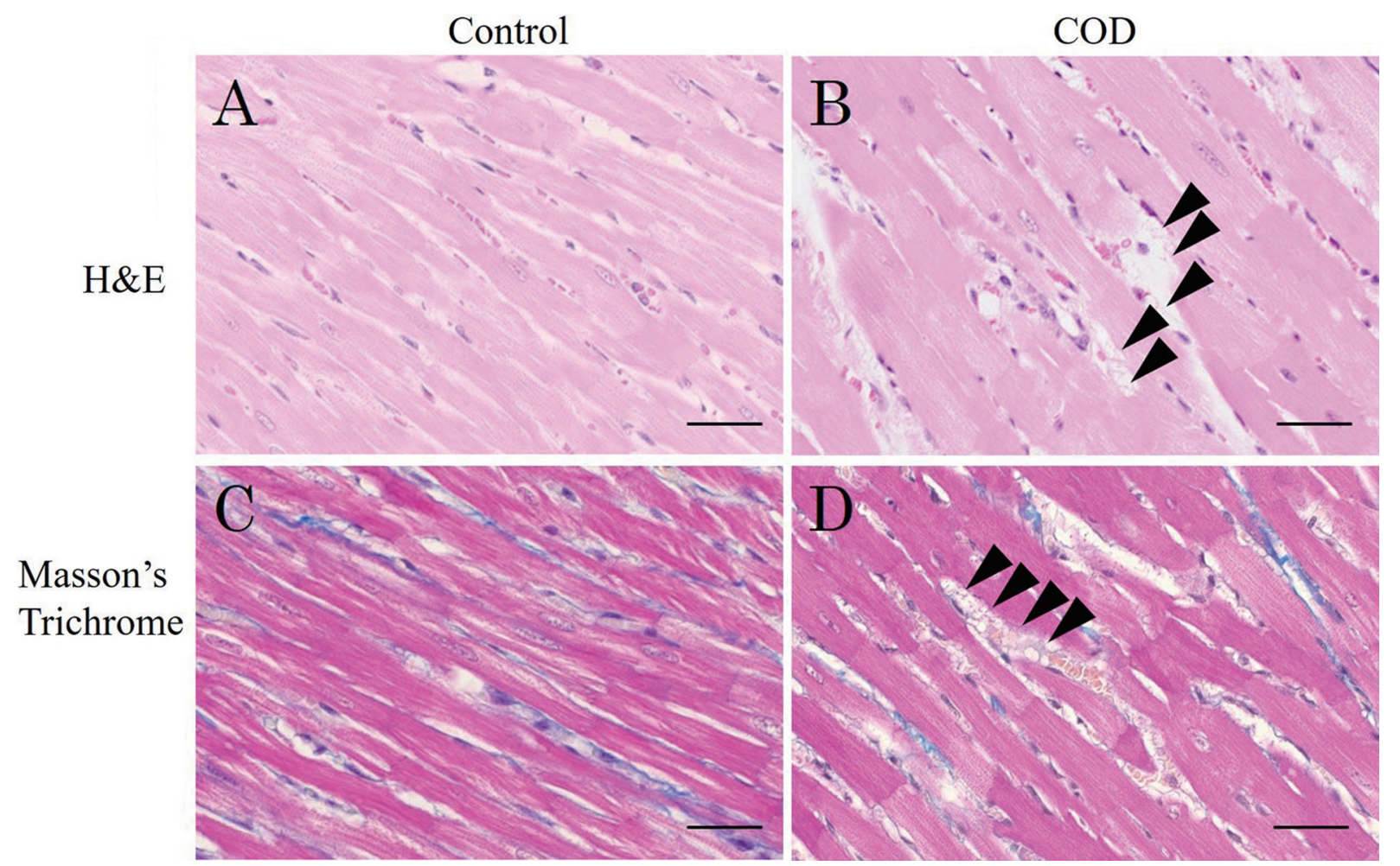

Fig. 2. Light microscopic images of hematoxylin and eosin (H\&E)- (A, B) and Masson's trichrome-stained (C, D) heart tissue sections from (A, C) control rats and (B, D) rats with copper deficiency (COD). A mesh-like structure (arrowheads) of capillary endothelial cells was observed within intact, non-degenerative myocardial tissue under the left ventricle in $(\mathrm{B}, \mathrm{D})$ the rats with $\mathrm{COD}$. Bar $=40 \mu \mathrm{m}$. 

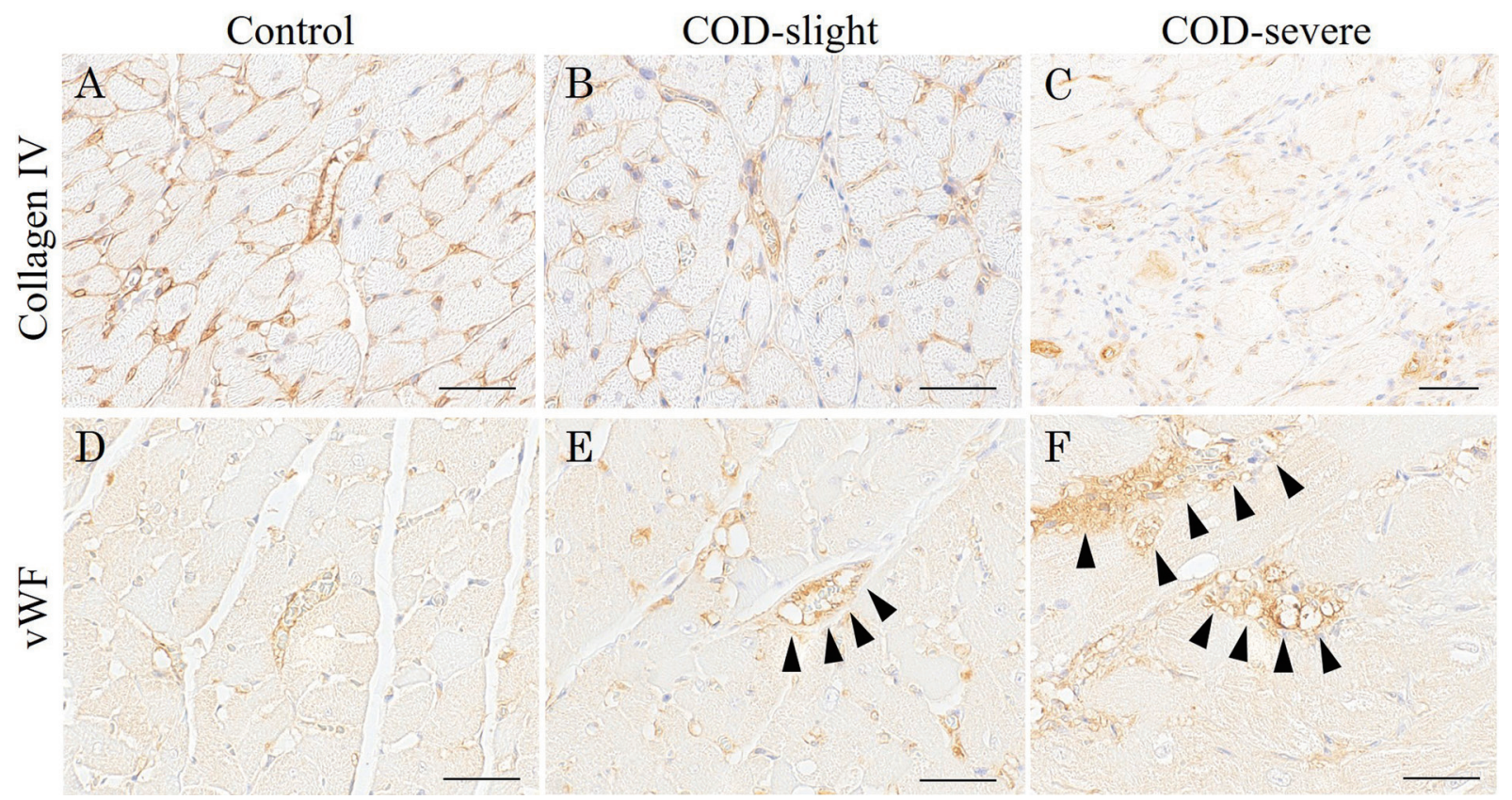

Fig. 3. Light microscopic images of immunohistochemically stained heart tissues from control rats and rats with copper deficiency (COD). (A) Most cardiomyocytes were labeled with collagen IV in the control rats. (B) Weak collagen IV-labeled cardiomyocytes were observed in the slight lesions of rats with COD. (C) No collagen IV-labeled cardiomyocytes were observed in the severe lesions of rats with COD. (A, B) $B a r=40 \mu \mathrm{m}$. (C) Bar $=50 \mu \mathrm{m}$. (D) von Willebrand factor (vWF)-labeled capillary endothelial cells in the control rats were smooth. (E) vWF-labeled capillary endothelial cells were spike-shaped in the lumen (arrowheads) of the slight lesions of rats with COD. (F) vWFlabeled capillary endothelial cells were thickened to form a mesh-like structure in the lumen (arrowheads) of the severe lesions in rats with COD. (D-F) Bar $=40 \mu \mathrm{m}$.
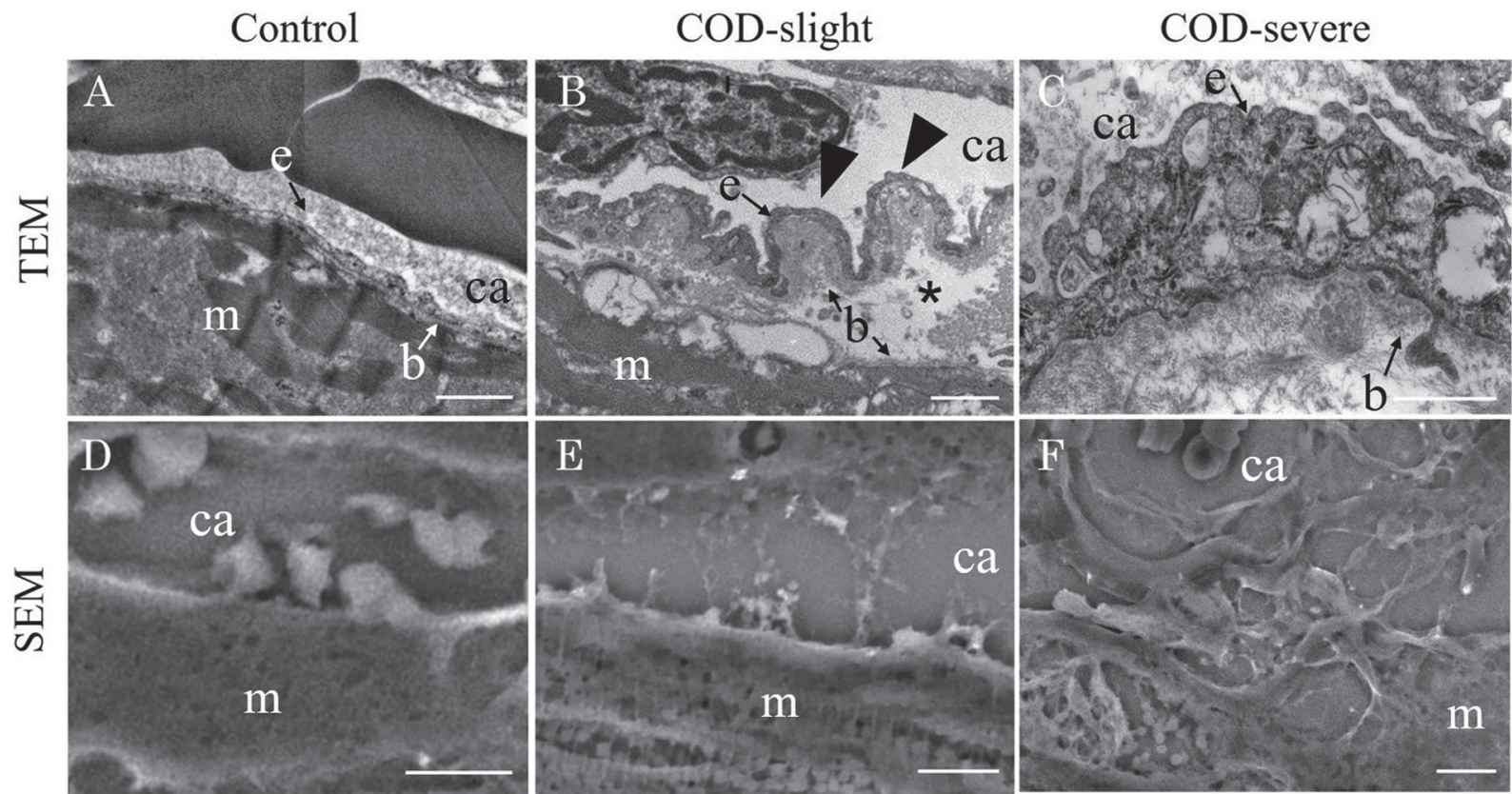

Fig. 4. Transmission and scanning electron micrographs of heart tissues from control rats and rats with copper deficiency (COD). (A) The basement membrane between the capillaries and the myocardial cells in the control rats was smooth. (B) The detachment of the basement membrane between the capillaries and myocardial cells (asterisk), and in part, the projections of the capillary endothelial cells into the lumen (arrowheads) in the slight lesions of rats with COD. (C) Thickened capillary endothelial cells, including the mitochondrial dilation in the severe lesions of rats with COD. (A-C) Bar $=1.0 \mu \mathrm{m}$. (D) The capillary endothelium surface in the control rats was smooth. The surface of the capillary endothelium was rough and protruding into the lumen in a (E) spike-like or (F) mesh-like manner in the (E) slight or (F) severe lesions of rats with COD. (D-F) Bar $=6.0 \mu \mathrm{m}$; ca, capillary; e, endothelial cell; b, basement membrane; m, myocardial cell. 
vascular endothelial cells and basement membranes correlated with each other, it is reasonable to assume that these changes are associated with a decrease in the survival of vascular endothelial cells due to basement membrane damage ${ }^{17}$. Furthermore, capillary compression due to myocardial contraction was stronger on the endocardial side than on the epicardium side ${ }^{18}$, and the focus was localized to just below the left ventricle with high ventricular pressure. Therefore, it is conceivable that a combination of mechanical and physical loads along with basement membrane damage could be an important characteristic of the pathogenesis.

Intimal hyperplasia with totipotent mesenchymal cells has been observed after a balloon-induced injury in the external carotid artery of copper-deficient rats ${ }^{19}$. Similarly, plexiform lesions observed in patients with pulmonary arterial hypertension are considered to be part of an overshooting regenerative process ${ }^{20}$. The network structure of vascular endothelial cells observed in this study may be explained as an abnormal neovascular proliferation process in the context of vascular endothelial damage. Moreover, the meshlike changes in vascular endothelial cells were observed within intact, non-degenerative myocardial tissue. These changes are likely to be early pathological changes, formed during the pathological regeneration process of the endothelial cells on the affected basement membranes. Furthermore, the pathogenesis of an abnormal neovascular proliferation may be related to decreased $\mathrm{Cu} / \mathrm{Zn}$-superoxide dismutase activity ${ }^{19}$ and excessive delivery of angiogenic factors ${ }^{21}$.

Mitochondrial dilation observed in the vascular endothelial cells is likely to be associated with a compensatory mechanism in response to the reduced activity of cytochrome c oxidase, a cuproenzyme in the electron transport system $^{22}$, or high metabolic demand of the myocardium during its adaptation to stress ${ }^{23}$. Therefore, the mitochondrial changes observed in vascular endothelial cells in this study may be associated with decreased cytochrome c oxidase activity or higher metabolic demand.

As the extent of vascular endothelial changes tended to be proportional to the extent of myocardial injury in our findings, the microcirculatory disturbance observed was likely related to abnormal vascular endothelial cell regeneration and may be associated with the occurrence of myocardial degeneration and necrosis. Furthermore, because mitochondrial changes and cardiomyocyte hypertrophy have been reported in a model of marginal dietary $\mathrm{COD}^{23}$, it follows that the initial changes in the myocardium leading to cardiac hypertrophy may include mitochondrial increase or swelling 22 . Subsequently, changes in intramyocardial capillaries are likely to induce microcirculatory disturbances, ultimately resulting in degeneration and necrosis of the myocardium. However, whether abnormal vascular remodeling causes progression in the pathology remains unclear ${ }^{20}$. Additional studies are needed to answer this question; including immunostaining with angiogenic and myocardial damage-related antibodies and additional measurement of biochemical myocardial markers.

It should be also added that these changes in the myo- cardium were distinguishable from those in progressive cardiomyopathy in rats in that they were stronger than those in the control and involved degenerative changes in the basement membrane and vascular endothelial cells.

In conclusion, mesh-like lesions of vascular endothelial cells were discovered adjacent to the left ventricular endocardium in the heart of rats with COD, likely caused by abnormal vascular remodeling after damage to the basement membrane and mechanical effects of myocardial contractions. Although cardiomyocyte damage may be related to microcirculatory disorders caused by damage to the basement membrane and vascular endothelial cells, additional investigations are necessary to better understand the exact mechanism of the pathogenesis.

Disclosure of Potential Conflicts of Interest: The authors declare no conflicts of interest.

Acknowledgments: The authors are grateful to Ren $\mathrm{Mu}-$ rakami and Shinji Ichikawa for their technical support.

\section{References}

1. Uauy R, Olivares M, and Gonzalez M. Essentiality of copper in humans. Am J Clin Nutr. 67(Suppl): 952S-959S. 1998.

2. Underwood EJ. Trace elements in human and animal nutrition, 4th ed. Academic Press, NewYork. 56-63. 2012.

3. Takagi Y. Encyclopedia of mineral, diseases, and minerals, 10: Intravenous and enteral nutrition. Yoshinori I (ed). Asakura Publishing, Tokyo. 651-680. 2003.

4. Klevay LM, Inman L, Johnson LK, Lawler M, Mahalko JR, Milne DB, Lukaski HC, Bolonchuk W, and Sandstead $\mathrm{HH}$. Increased cholesterol in plasma in a young man during experimental copper depletion. Metabolism. 33: 1112-1118. 1984.

5. Reiser S, Smith JC Jr, Mertz W, Holbrook JT, Scholfield DJ, Powell AS, Canfield WK, and Canary JJ. Indices of copper status in humans consuming a typical American diet containing either fructose or starch. Am J Clin Nutr. 42: 242-251. 1985.

6. Shields GS, Coulson WF, Kimball DA, Carnes WH, Cartwright GE, and Wintrobe MM. Studies on copper metabolism. 32. Cardiovascular lesions in copper-deficient swine. Am J Pathol. 41: 603-621. 1962.

7. Kelly WA, Kesterson JW, and Carlton WW. Myocardial lesions in the offspring of female rats fed a copper deficient diet. Exp Mol Pathol. 20: 40-56. 1974.

8. Medeiros DM, Bagby D, Ovecka G, and McCormick R. Myofibrillar, mitochondrial and valvular morphological alterations in cardiac hypertrophy among copper-deficient rats. J Nutr. 121: 815-824. 1991.

9. Allen KG, and Klevay LM. Cholesterolemia and cardiovascular abnormalities in rats caused by copper deficiency. Atherosclerosis. 29: 81-93. 1978.

10. Viestenz KE, and Klevay LM. A randomized trial of copper therapy in rats with electrocardiographic abnormalities due to copper deficiency. Am J Clin Nutr. 35: 258-266. 1982.

11. Davidson J, Medeiros DM, and Hamlin RL. Cardiac ultra- 
structural and electrophysiological abnormalities in postweanling copper-restricted and copper-repleted rats in the absence of hypertrophy. J Nutr. 122: 1566-1575. 1992.

12. DiNicolantonio JJ, Mangan D, and O'Keefe JH. Copper deficiency may be a leading cause of ischaemic heart disease. Open Heart. 5: e000784. 2018.

13. Farquharson $\mathrm{C}$, and Robins SP. Immunolocalization of collagen types I, III and IV, elastin and fibronectin within the heart of normal and copper-deficient rats. J Comp Pathol. 104: 245-255. 1991.

14. Medeiros DM, Davidson J, and Jenkins JE. A unified perspective on copper deficiency and cardiomyopathy. Proc Soc Exp Biol Med. 203: 262-273. 1993.

15. Vracko R, and Benditt EP. Basal lamina: the scaffold for orderly cell replacement. Observations on regeneration of injured skeletal muscle fibers and capillaries. J Cell Biol. 55: 406-419. 1972.

16. Suma K. A study on ventricular working condition under pressure overload by arterial stenosis, concept and significance of steady-state maximum ventricular pressure and maximum pressure difference. Heart. 4: 826-836. 1972.

17. Shibutani J. General animal pathology, 3rd ed, 5: Cell proliferation and differentiation and their abnormalities, 3 :
Extracellular matrix. The Japanese Society of Veterinary Pathology (ed). Bun-eido Publishing, Tokyo. 77-82. 2001.

18. Goto M. Microcirculation of the heart. B\&R. 17: 11-17. 2003.

19. Dalle Lucca JJ, Saari JT, Falcone JC, and Schuschke DA. Neointima formation in the rat carotid artery is exacerbated by dietary copper deficiency. Exp Biol Med (Maywood). 227: 487-491. 2002.

20. Jonigk D, Golpon H, Bockmeyer CL, Maegel L, Hoeper MM, Gottlieb J, Nickel N, Hussein K, Maus U, Lehmann U, Janciauskiene S, Welte T, Haverich A, Rische J, Kreipe $\mathrm{H}$, and Laenger $\mathrm{F}$. Plexiform lesions in pulmonary arterial hypertension composition, architecture, and microenvironment. Am J Pathol. 179: 167-179. 2011.

21. Hsieh PC, Davis ME, Lisowski LK, and Lee RT. Endothelial-cardiomyocyte interactions in cardiac development and repair. Annu Rev Physiol. 68: 51-66. 2006.

22. Mao S, Medeiros DM, and Wildman RE. Cardiac hypertrophy in copper-deficient rats is owing to increased mitochondria. Biol Trace Elem Res. 64: 175-184. 1998.

23. Li Y, Wang L, Schuschke DA, Zhou Z, Saari JT, and Kang YJ. Marginal dietary copper restriction induces cardiomyopathy in rats. J Nutr. 135: 2130-2136. 2005. 